\title{
Characterizing Optical Properties of Self-Assembled Gold Nanoparticles for Surface Plasmon Resonance Device Applications
}

\author{
Hsuen-Li CHEN*, Hsu-Chun CHENG ${ }^{1}$, Tsung-Shine Ko ${ }^{1}$, Shang-Yu ChuANg and Tien-Chi $\mathrm{CHU}^{2}$ \\ Department of Materials Science and Engineering, National Taiwan University, Taipei, Taiwan, R.O.C. \\ ${ }^{1}$ National Nano Device Laboratry, Hsinchu, Taiwan, R.O.C. \\ ${ }^{2}$ Yuanpei Institute of Science and Technology, Hsinchu, Taiwan, R.O.C.
}

(Received December 5, 2005; revised February 27, 2006; accepted May 17, 2006; published online September 7, 2006)

In this study, the optical constants of gold nanoparticles are evaluated for surface plasmon-based sensor applications. Using an effective medium approximation (EMA) and ellipsometry, approaches to monitor the self-assembly of gold nanoparticles are also demonstrated. Spectroscopic ellipsometric parameters measured $(\tan \Psi, \cos \Delta)$ before and after adding gold nanoparticles to a substrate are used to calculate the optical constants of gold nanoparticles. The film thickness is measured by grazing incidence X-ray reflectivity (XRR). The optical constants (refractive index, extinction coefficient) of gold nanoparticles can be obtained from the measured ellipsometric parameters and thickness. We also show that particles density can be well predicted and detected nondestructively by this method. [DOI: 10.1143/JJAP.45.6984]

KEYWORDS: gold-nanoparticles, self-assembly, optical constants, surface plasmon resonance, ellipsometric parameters

Nanoscale particles have recently received much attention owing to their special characteristics and can possibly be used in electronic devices, photonics, catalysis, and biochips. To control self-assembly well, characterize the optical properties of nanoparticles and accurately predict the behaviors of optical devices, a simple and nondestructive method for monitoring self-assembly is required. In general, $\mathrm{UV} /$ visible transmission spectra and definition transmission electron microscopy (TEM) images are used to determine the size and morphology of nanoparticles. However, TEM has a complicated and destructive operation procedure. The accuracy of UV/visible transmission spectra for characterizing gold nanoparticles is not sufficient and a high-transmission substrate is required for measuring transmission spectra. In this study, we evaluate the self-assembly status and optical properties of gold nanoparticles by ellipsometry and X-ray reflectivity (XRR).

Surface plasmon resonance (SPR) is extensively used in chemical and biological sensors. ${ }^{1)}$ In general, SPR involves the optical excitation of surface plasmon waves on a gold or silver film employing the Kretschmann configuration for attenuated total reflection (ATR), as shown in Fig. 1. ${ }^{2)}$ Reflectivity is measured as a function of the incidence angle $\theta$ of the light beam. The ATR experiment has a reflectivity minimum at the initial angle in an angle-scanned SPR instrument. The applications of an SPR-based sensor are related to the concentration and chemical properties of the sample. It has been demonstrated that incorporating gold nanoparticles can considerably enhance the sensitivity of SPR sensors. ${ }^{3,4)}$ In this method, a self-assembled monolayer (SAM) of a dithiol is formed on the substrate, and a nanoparticle layer is linked to the SAM. ${ }^{5)}$ The effects of colloidal gold on ATR curves are shifted plasmon angles, broadened curves and increased minimum reflectivities; these changes reflect the modulation of the surface plasmon dispersion relationship. For this reason, to control selfassembly well and understand the optical properties of nanoparticles to accurately predict the behaviors of optical devices, characterizing the optical properties of self-assembled gold nanoparticles is important.

*E-mail address: hsuenlichen@ntu.edu.tw

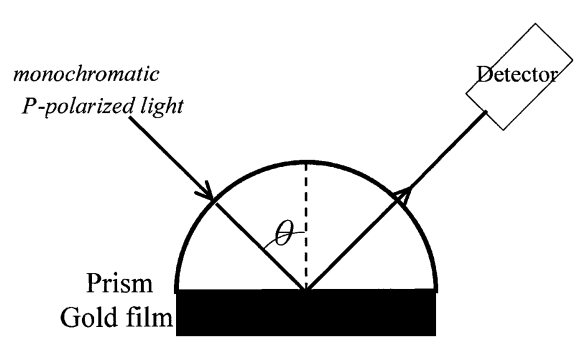

Fig. 1. Diagram of surface plasmons resonance device enhanced by gold nanoparticles.

The complex refractive index $N=(n-\mathrm{i} k)$ is an important parameter for understanding both the optical and electrical properties of gold nanoparticles, where $n$ is the refractive index and $k$ is the extinction coefficient. In this study, we regard gold nanoparticles as a thin film on the basis of the effective medium theory ${ }^{6)}$ and deposited gold nanoparticles for different durations on SAMs to find the saturation density and duration of gold nanoparticles. After this, we measured layer thickness by X-ray reflectivity and scanning electron microscopy (SEM). Then we measured ellipsometric parameters ( $\tan \Psi, \cos \Delta$ ) using an ellipsometer to calculate the optical constants of gold nanoparticles. To confirm the degree of accuracy of this approach, we used the optical constants of gold nanoparticle film to estimate thickness and porosity. Finally, we simulated and obtained the optimized thickness of gold film for SPR measurement.

All chemical materials were of guarantee reagent (G.R.) grade. Sodium citrate and hydrogen tetrachloroaurate $\left(\mathrm{HAuCl}_{4}\right)$ were purchased from Tokyo Chemical Industries. Colloidal gold solution was prepared by the sodium citrate reduction of $\mathrm{HAuCl}_{4}$ under boiling conditions. The silicon substrates were cleaned and then immersed into a solution of 3-aminopropyl-trimethoxysilan (ATPMS) in ethanol to form the SAM. The ATPMS-covered silicon substrates were rinsed with ethanol and deionized water and then immersed into a solution for the citrate reduction of $\mathrm{HAuCl}_{4}$ for 10 to $50 \mathrm{~min}$. Finally, the gold nanoparticles attached substrates were washed and dried for SEM, XRR, and ellipsometer measurement. SEM (JEOL JSM-6500F) was used for observing the morphologies and thickness of gold nano- 


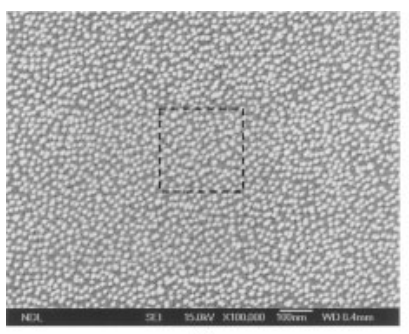

(a)

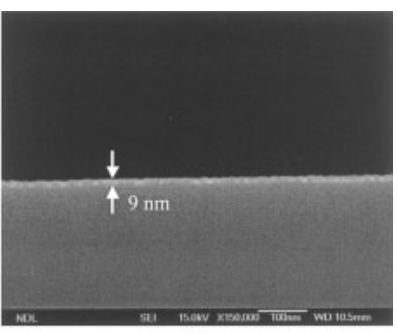

(b)
Fig. 2. (a) Top view and (b) cross-section SEM images of gold nanoparticle film.

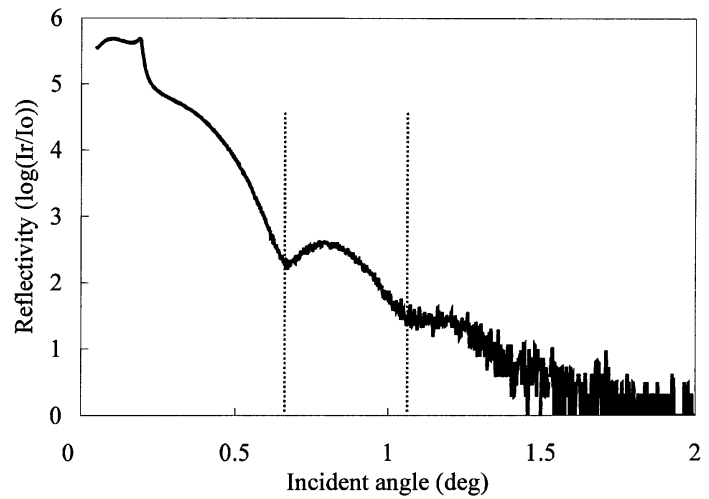

Fig. 3. Grazing incidence XRR spectrum of gold nanoparticle film.

particle films. XRR (PHILIPS X'Pert Pro) was used to calculate the thickness of gold nanoparticle films. We measured samples to obtain ellipsometric angles, $\Psi$ and $\Delta$, using an ellipsometer (SOPRA SE5) in the wavelength range from 300 to $800 \mathrm{~nm}$. The ellipsometric data were fitted using a classical Lorentz oscillator model calculation for optical constants. On the basis of previous results, we simulated the optical behavior of the gold-nanoparticle-modified SPR devices utilizing the optical-thin-film theory. ${ }^{7)}$

Figure 2 shows SEM images of the gold nanoparticles in our experiments. Figure 2(a) shows the surface morphology of gold nanoparticles linked on functionalized silicon substrates. The gold nanoparticles are spread uniformly and mostly have the same diameter. In this study, we treated the gold nanoparticles as an optical thin film. Figure 2(b) shows a cross-sectional image of gold nanoparticles. Figure 1 indicates that the gold nanoparticles are dispersed uniformly without large-scale aggregation on the surface. The average diameter of gold nanoparticle film is estimated to be $9.0 \mathrm{~nm}$. As shown in Fig. 3, we also measured film thickness by grazing incidence $\mathrm{X}$-ray reflectivity. The thickness of the nanoparticle film calculated by the Born approximation is $9.1 \mathrm{~nm}$, which is comparable to the thickness in the SEM image as shown in Fig. 2(b).

For calculating optical constants of gold nanoparticle film, we measured the ellipsometric parameters using a spectroscopic ellipsometer. Figure 4 shows the ellipsometric parameters $(\tan \Psi, \cos \Delta)$ of gold nanoparticle films coated on silicon substrate with and without adding an APTMS layer. We found that the APTMS layer did not significantly affect the ellipsometric curves but the gold nanoparticles did. We could also monitor the self-assembly of gold nanoparticles

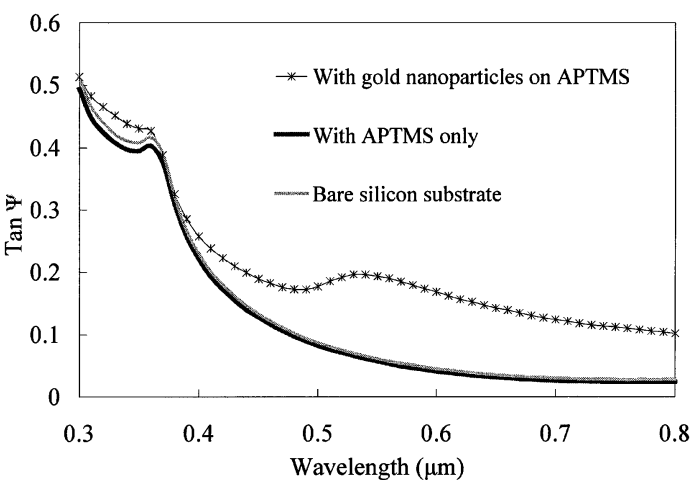

(a)

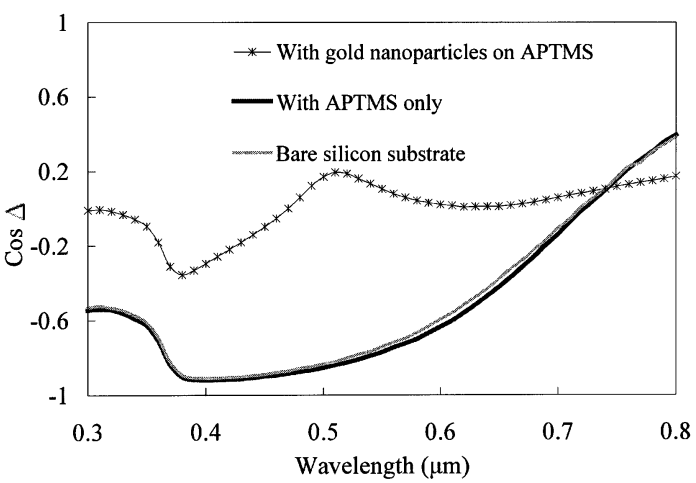

(b)

Fig. 4. The measured spectroscopic ellipsometric parameters (a) $\tan \Psi$ and (b) $\cos \Delta$ of gold nanoparticle films with different deposition durations.

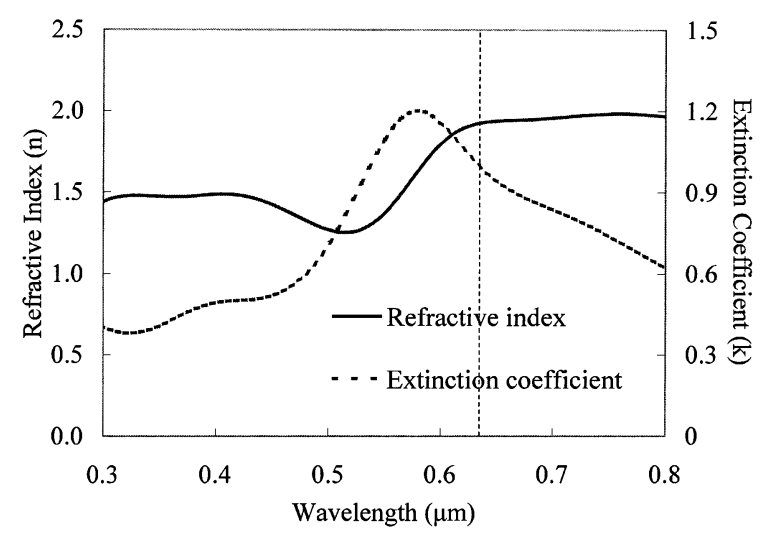

Fig. 5. Optical constants of gold nanoparticle film.

by this nondestructive method.

The optical constants of gold nanoparticle film were calculated by a curve fitting method based on the Lorentz oscillator model. The optical constants of gold nanoparticle film are shown in Fig. 5. There is an absorption band located in the range from 500 to $600 \mathrm{~nm}$ in the extinction coefficient curve, which is attributed to the plasmon absorption band of the gold nanoparticles. The peak wavelength $(580 \mathrm{~nm})$ of the plasmon absorption band is larger than that of the same gold nanoparticles in solution $(520 \mathrm{~nm})$. This appearance of redshift resulted from the aggregation of nanoparticles in the silicon substrate.

Figure 6(a) shows an SEM image of gold nanoparticles 


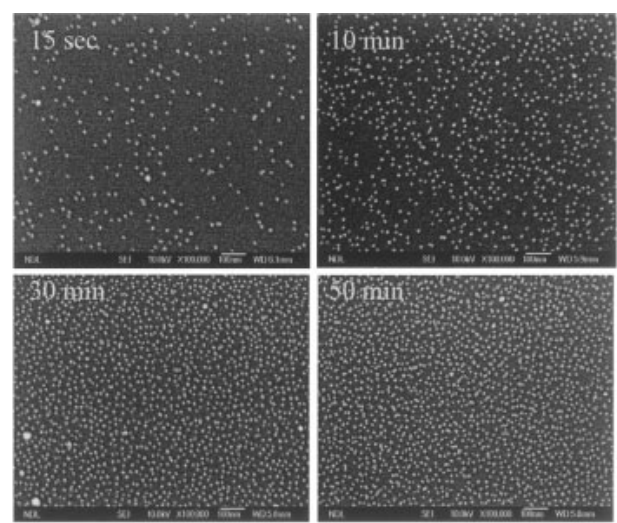

(a)

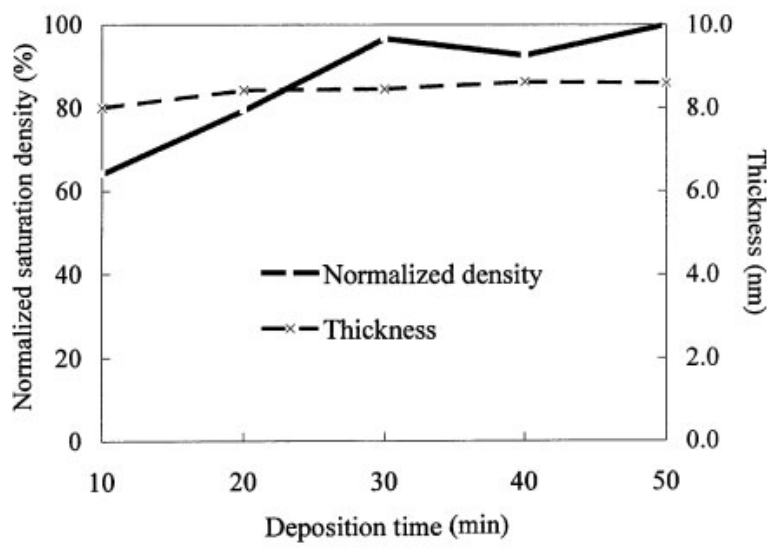

(b)

Fig. 6. (a) SEM images of gold nanoparticle film with different deposition durations. (b) Thickness and density of gold nanoparticle film with different deposition durations.

self-assembled on a silicon substrate. The density of gold particles increased with increasing self-assembly duration. We also found that the gold particles were uniformly spread on the substrate. Here, we evaluated the density and thickness of gold nanoparticle film with different deposition durations by the effective medium approximation (EMA) approach. From Fig. 6(b), the thicknesses of gold nanoparticle film with different deposition durations were slightly different. The intensity was increased from 64 to $100 \%$ with deposition durations and the saturation duration of gold nanoparticle film was about $30-50 \mathrm{~min}$. This result strongly supports the theory that the gold nanoparticles deposit in the monolayer. Therefore, we can monitor the self-assembly by this method. Furthermore, a He-Ne laser $(\lambda=632.8 \mathrm{~nm})$ was generally applied in an SPR device as a light source owing to its suitable wavelength, intensity and stability. As shown in Fig. 5, the optical constants of gold nanoparticle film at $632.8 \mathrm{~nm}$ are $(1.925,1.002)$.

It is possible under certain experimental conditions to considerably enhance the sensitivity of a conventional SPR device by using gold nanoparticles. The SPR curves of the gold nanoparticles for ATR, which was simulated for a 50$\mathrm{nm}$-thick gold film and $10 \mathrm{~nm}$ Gold nanoparticles. We found that upon adding gold nanoparticles on gold film, the SPR curve broadened and the minimum reflectance increased from less than 1 to $20 \%$. To enhance the sensitivity of the gold-nanoparticle-based SPR sensor, we must find a suitable

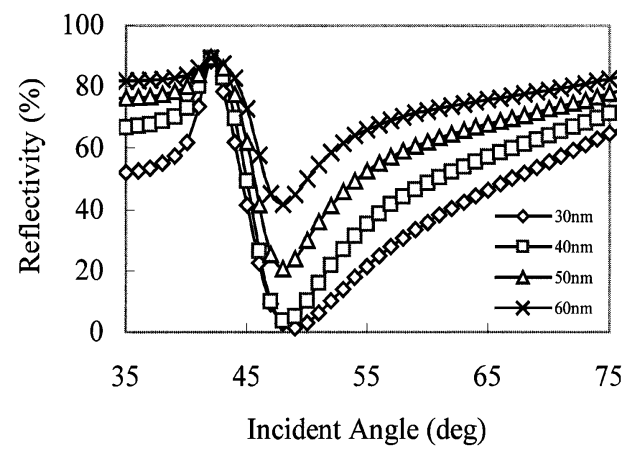

Fig. 7. Simulated SPR curves of gold-nanoparticles-based sensors with varying thicknesses of underlying gold films.

thickness of the underlying gold film. Figure 7 shows the simulated thicknesses of the underlying gold film from 30 to $60 \mathrm{~nm}$. It is apparent that when the thickness of the underlying gold film is about $30-40 \mathrm{~nm}$, the SPR curve is sharper than the others and also has a more apparent reflection dip.

In this study, we evaluated the optical constants of gold nanoparticles for surface plasmon-based sensors applications. The gold nanoparticles were uniformly spread on a silicon substrate and treated as an optical thin film. We measured the spectroscopic ellipsometric parameters ( $\tan \Psi, \cos \Delta$ ) before and after adding gold nanoparticles to calculate the optical constants of gold nanoparticles. Before a classical Lorentz oscillator model calculation, we measured film thickness by grazing incidence XRR. The thickness of the nanoparticle film calculated using the Born approximation was $9.1 \mathrm{~nm}$, which is consistent with the thickness observed in the SEM image. The optical constants (refractive index, extinction coefficient) of gold nanoparticles could be obtained from the measured ellipsometric parameters and thickness. The measured optical constants of gold nanoparticle film at $632.8 \mathrm{~nm}$ were $(1.925,1.002)$. It was found that the nanoparticle films show unique optical properties different from those of bulk gold film, and that it is possible to control the self-assembly by monitoring film density using the effective medium approximation approach. Furthermore, it was found that we can obtain the optical constants of various nanoparticles employed in the procedures and that the optimal thickness of the underlying gold film is about $30-40 \mathrm{~nm}$ for linking the gold nanoparticles.

The authors are very thankful to the National Science Council, Taiwan, R.O.C. for supporting this study under the projects NSC-94-2215-E-002-026 and NSC-94-2216-E-002022.

1) R. L. Rich and D. G. Myszka: Curr. Opin. Biotechnol. 11 (2000) 54.

2) K. A. Peterlinz and R. Georgiadis: Langmuir 12 (1996) 4731.

3) L. A. Lyon, D. J. Pena and M. J. Natan: J. Phys. Chem. B 103 (1999) 5826.

4) L. A. Lyon, M. D. Musick and M. J. Natan: Anal. Chem. 70 (1998) 5177.

5) T. Baum, D. Bethell, M. Brust and D. J. Schiffrin: Langmuir 15 (1999) 866.

6) L. Ward: The Optical Constants of Bulk Materials and Films (IOP Publishing, Bristol, 1994) 2nd ed.

7) R. M. A. Azzam and N. M. Bashara: Ellipsometry and Polarized Light (North-Holland, Amsterdam, 1977). 